\title{
Combination Therapies of Radioembolization and Systemic Agents for Primary and Secondary Liver Tumors
}

\author{
Ryan M. Hickey, MD, FSIR ${ }^{1}$ \\ ${ }^{1}$ Division of Vascular Interventional Radiology, Department of \\ Radiology, NYU Langone, NYU Grossman School of Medicine, New \\ York, New York
}

Dig Dis Interv 2021;5:148-152.

\begin{abstract}
Address for correspondence Ryan M. Hickey, MD, FSIR, Division of Vascular Interventional Radiology, Department of Radiology, NYU Langone, NYU Grossman School of Medicine, 660 First Avenue, Suite 300, New York, NY 10016 (e-mail: ryan.hickey@nyulangone.org).
\end{abstract}
Abstract Keywords
- radioembolization
- systemic therapy
- chemotherapy
- Y-90

This review provides an overview of studies in which radioembolization was combined with systemic agent(s). Several reports in the literature provide retrospective evaluation of the use of concomitant radioembolization with systemic agents; however, in an effort to limit the scope of this review to the highest levels of evidence available, the studies discussed are restricted to prospective phase 2 and 3 clinical trials of combination therapy.

The benefit of combining radioembolization with systemic agents for the treatment of primary and metastatic liver tumors has long been postulated, whether as an opportunity to modulate changes in tumor signaling pathways induced by radiation, to take advantage of the radiosensitizing effects of several chemotherapeutic agents, or, in the era of immunotherapy, to trigger an immune response that might increase the effectiveness of checkpoint-inhibitor immunotherapy.

This review provides an overview of studies in which radioembolization was combined with systemic agent(s). Several reports in the literature provide retrospective evaluation of the use of concomitant radioembolization with systemic agents; however, in an effort to limit the scope of this review to the highest levels of evidence available, the studies discussed are restricted to prospective phase 2 and 3 clinical trials of combination therapy.

\section{Hepatocellular Carcinoma}

\section{Combination Therapy with Sorafenib as a Bridge to Liver Transplantation}

In 2014, Kulik et al reported the outcomes of patients with HCC treated with radioembolization with or without sorafenib as a bridge to liver transplantation. ${ }^{1}$ Twenty patients with HCC who were considered potential liver transplanta-

received

December 23, 2020 accepted after revision March 15, 2021
Issue Theme Y-90; Guest Editors, Ripal T. Gandhi, MD, FSIR, FSVM and Suvranu Ganguli, MD, FSIR

tion candidates (up to UCSF criteria, i.e., solitary tumor $\leq 6.5 \mathrm{~cm}$, or $\leq 3$ nodules, the largest being $\leq 4.5 \mathrm{~cm}$ and the sum of tumor diameters $\left.\leq 8 \mathrm{~cm}^{2}\right)^{2}$ with Child-Pugh (C-P) scores $\leq \mathrm{B} 8$ were randomized to receive radioembolization alone or in combination with sorafenib. Patients in both arms received radioembolization with glass microspheres. Patients in the combination therapy arm received sorafenib for a median of 20 days prior to radioembolization with no interruption of sorafenib therapy for radioembolization.

All patients in the combination arm required dose reduction, the majority of which were due to dermatologic side effects. One patient, who was also taking hepatitis $C$ medications, developed grade 4 bilirubin toxicity. Complete discontinuation of sorafenib due to side effects occurred in $50 \%$ of patients.

Seventeen of the patients (85\%) received liver transplantation ( 9 patients in the control arm, 8 patients in the combination therapy arm). There were no significant differences in the tumor treatment responses between the two groups based on explant pathological analyses, with 7, 1, and 2 tumors in the radioembolization-only arm, and 4, 2, and 2 tumors in the combination arm exhibiting 100 , more than 50 , and less than $50 \%$ nonviable tissue, respectively $(p=0.69)$.

With regard to posttransplant complications, four patients (50\%) who received combination therapy developed

(c) 2021. Thieme. All rights reserved. Thieme Medical Publishers, Inc., 333 Seventh Avenue, 18th Floor, New York, NY 10001, USA
DOI https://doi.org/ 10.1055/s-0041-1729950. ISSN 2472-8721. 
biliary anastomotic complications (three strictures, one leak + stricture) within 30 days of liver transplantation. There were no biliary complications in the control group. Three of the patients in the combination therapy group (37.5\%) developed acute cellular rejection, whereas no patients in the control group developed rejection.

The authors concluded that the equivalent response rates between the two groups combined with the higher rate of adverse events among the patients receiving combination therapy supports continued use of locoregional therapy alone as a bridge to liver transplantation.

\section{Combination Therapy with Sorafenib for Advanced HCC}

In 2019, Ricke et al reported the outcomes of combination radioembolization with sorafenib for patients with advanced HCC from the SORAMIC trial. ${ }^{3}$ Patients with preserved liver function (C-P scores $\leq$ B7) and HCC Barcelona Clinic Liver Cancer (BCLC) stage C, or BCLC stage B disease not eligible for transarterial chemoembolization (TACE), were considered eligible. BCLC C patients with extrahepatic disease were permitted as long as the HCC was liver dominant and did not present with pulmonary metastases.

A total of 216 patients were randomized to the control arm of sorafenib only and 208 were randomized to the investigational arm to receive combination radioembolization and sorafenib. In the combination therapy group, radioembolization was performed with resin microspheres a median of 22 days following randomization, and sorafenib was initiated a median of 3 days after completion of radioembolization. In the control arm, sorafenib was initiated a median of 4 days following randomization. Ultimately 114 patients in the investigational arm received combination therapy according to the study protocol, while 174 patients in the control arm received sorafenib therapy according to the study protocol.

The intention-to-treat (ITT) analysis demonstrated a median overall survival (OS) of 12.1 months (95\% confidence interval [CI] 10.7-14.9) in the combination therapy group compared with 11.4 months (95\% CI: 9.9-14.0) in the sorafenib-only group (hazard ratio [HR]: 1.01 ; $95 \% \mathrm{CI}$ : $0.81-1.25$; $p=0.953$ ). Given the high number of patients who did not complete the study according to protocol, a per-protocol analysis was also reported. In the per-protocol analysis, median OS was 14.0-months (95\% CI: 11.5-17.0) in the combination therapy group, and 11.1-months (95\% CI: 9.8-13.8) in the sorafenib-only group (HR: 0.86 ; 95\% CI: $0.67-1.11 ; p=0.252$ ).

Subgroup analysis of the per-protocol population suggested a survival benefit for patients $\leq 65$ years old (HR: 0.65 ; 95\% CI: $0.43-1.00 ; p-=-0.046)$, noncirrhotic patients (HR: $0.46 ; 95 \% \mathrm{CI}: 0.25-0.86 ; p=0.013$ ), and patients with HCC of nonalcoholic etiology (HR: $0.63 ; 95 \% \mathrm{CI}$ : $0.45-0.89$; $p=0.009$ ).

Grade 3-4 adverse events were reported in $64.8 \%$ of patients in the combination therapy arm and 53.3\% of patients in the control arm $(p=0.036)$. Of note, hyperbilirubinemia of grades 3 to 4 was approximately four times more common in the combination therapy arm (8.8 vs. $2.2 \%$, $p=0.007)$, although the authors reported the hepatobiliary adverse events to be in line with previously reported adverse events of radioembolization. Grade 5 adverse events (i.e., patient death) occurred in two patients (1.3\%) in the combination therapy arm, and in two patients in the control arm (1.0\%).

The authors cited several factors that may have led to the failure of the study to demonstrate a benefit from combination therapy, including the breadth of the patient population recruited for the study, particularly patients with compromised liver function, and suboptimal radioembolization dosimetry. Patients with higher C-P scores had a higher rate of protocol deviations in the combination therapy arm, most often due to failure to undergo a second radioembolization procedure and/or missing the initiation of sorafenib therapy. Protocol deviations confounded the ITT analysis, and the smaller number of patients remaining for the per-protocol analysis may have caused this analysis to be underpowered to demonstrate a difference between the two study arms.

With regard to the suboptimal radioembolization dosimetry, absorbed tumor dose thresholds have been shown to correlate with survival. ${ }^{4,5}$ However, such tumor dose thresholds were not included in the SORAMIC protocol and may have limited the efficacy of the radioembolization techniques under study.

\section{Newer Systemic Agents for HCC}

The treatment paradigm for advanced HCC has changed drastically over the past several years. After nearly 10 years without an effective systemic treatment option other than sorafenib, between 2017 and 2020, eight new systemic therapies have been approved by the Food and Drug Administration (FDA) for the treatment of advanced HCC as either first- or second-line therapy, including regorafenib (2017), nivolumab (2017), lenvatinib (2018), pembrolizumab (2018), cabozantinib (2019), ramucirumab (2019), nivolumab + ipilimumab (2020), and atezolizumab + bevacizumab (2020), with lenvatinib and atezolizumab + bevacizumab being the only therapies approved for first-line treatment.

There are several clinical trials underway investigating the use of radioembolization with these systemic therapies, particularly the checkpoint-inhibitor immunotherapies (nivolumab, pembrolizumab, ipilimumab); however, the only reports of combination therapy with radioembolization and these new agents to date are limited to a retrospective study of the safety of concurrent radioembolization and immunotherapy. ${ }^{6}$

\section{Intrahepatic Cholangiocarcinoma}

Edeline et al published in 2019 the results of the MISPHEC trial, a multicenter phase 2 clinical trial investigating the combination of glass microsphere radioembolization to firstline chemotherapy for unresectable intrahepatic cholangiocarcinoma. ${ }^{7}$ Forty-one patients received concomitant gemcitabine and cisplatin chemotherapy. For patients with unilobar disease, radioembolization was performed during 
cycle 1 , whereas patients with bilobar disease received radioembolization during chemotherapy cycles 1 and 3 . The gemcitabine dose was reduced for the cycle concomitant and the cycle following radioembolization. The primary endpoint was 3-month response rate, with secondary endpoints evaluating toxicity, progression-free survival (PFS), OS, disease control rate, and response rate according to the Choi criteria. The Choi criteria, originally developed to assess the treatment response of gastrointestinal tumors, have been subsequently applied to several different tumor types. The Choi criteria use the changes in tumor dimensions and target lesion density to determine response, defining a partial response rate as $\geq 10 \%$ decrease in the sum of largest tumor diameter or $\geq 15 \%$ decrease in the mean of tumor density. ${ }^{8}$

The primary endpoint demonstrated a response rate according to RECIST criteria was 39\% (90\% CI: $26-53 \%)$. Secondary endpoints indicated a 3-month disease control rate of $98 \%$ (95\% CI: 89-99\%). and a Choi response rate of 93\% (95\% CI: 82-98\%). The median PFS was 14 months (95\% CI; 8-17 months) with a median OS of 22 months (95\% CI: $14-52$ months). Nine patients (22\%) were downstaged to surgical resection, of whom 8 patients received an $\mathrm{R} 0$ resection. Two patients who could not undergo liver resection did receive liver transplantation. Each of these patients developed recurrence posttransplantation in the form of a solitary pulmonary nodule.

Toxicities in the trial were most often related to chemotherapy-related toxicities, including grade 3 to 4 hematologic toxicities of which neutropenia was the most common (51\%). Hepatic toxicities were more common in patients with cirrhosis. Nine of the twelve patients (75\%) with cirrhosis developed hepatic failure (characterized as any grade of ascites or jaundice), of which five were nonreversible. Five of 29 patients (17\%) without cirrhosis developed hepatic failure, all of which were reversible. All of the patients who developed nonreversible hepatic failure had received wholeliver radioembolization.

The authors highlighted the benefit of combination of radioembolization and chemotherapy as a downstaging option to potentially curative resection in patients otherwise considered only for palliative treatment. However, the authors recommended this strategy of combination of chemotherapy and radioembolization be avoided in patients with cirrhosis due to the high rates of hepatic failure among patients with cirrhosis.

A phase 3 study evaluating the addition of resin microsphere radioembolization to chemotherapy consisting of gemcitabine and cisplatin is currently underway (SIRT Followed by CIS-GEM Chemotherapy vs CIS-GEM Chemotherapy Alone as First Line Treatment of Patients with Unresectable Intrahepatic Cholangiocarcinoma [SIRCCA]). The study is estimated to complete in June 2021.

\section{Metastatic Colorectal Cancer}

\section{Radioembolization with Hepatic Artery Chemotherapy}

Gray et al reported the outcomes for patients with hepatic colorectal metastases treated with resin microsphere radio- embolization plus hepatic artery chemotherapy (HAC) compared with HAC alone in 2001. ${ }^{9}$

Seventy-four patients with bilobar metastatic colorectal cancer limited to the liver underwent laparotomy, cholecystectomy, and insertion of a permanent hepatic artery catheter attached to a subcutaneous access port for intra-HAC. The patients were randomized to receive radioembolization plus continuous infusion floxuridine chemotherapy, or continuous infusion floxuridine alone. The study included patients who were receiving first-line chemotherapy as well as patients who had previously received palliative chemotherapy.

After four patients had been deemed ineligible after randomization, and one patient died prior to any protocol treatment, 35 patients received radioembolization plus HAC and 34 patients received HAC. Resin microsphere radioembolization was performed as a single injection of microspheres via the subcutaneous access port.

Patients who received combination therapy had greater response rates ( $44 \mathrm{vs.} 17.6 \%, p=0.01$ ) and a greater reduction in carcinoembryonic antigen (CEA) levels (72 vs. 47\%, $p=0.004$ ) than the patients who received HAC alone. In addition, the median time to progression (TTP) in the liver was longer in the investigational arm compared with that in the control arm based on tumor area (15.9 vs. 9.7 months, $p=0.001$ ), tumor volume (12.0 vs. 7.6 months, $p=0.04)$, or CEA (6.7 vs. 5.7 months, $p=0.06$ ). There was no difference in OS between the two groups. The findings led to FDA approval of resin microsphere for the treatment of unresectable hepatic metastases of colorectal cancer with adjuvant intra-HAC with floxuridine.

\section{Radioembolization with Systemic Intravenous Chemotherapy}

In 2004, Van Hazel et al published a study on the outcomes of patients with previously untreated, unresectable hepatic colorectal metastases with fluorouracil/leucovorin chemotherapy with or without resin microsphere radioembolization. ${ }^{10}$ Twenty-one patients were randomized, of whom 11 received the combination therapy and 10 received chemotherapy alone. Time to tumor progression was longer for patients receiving combination therapy compared with chemotherapy alone (18.6 vs. 3.6 months, $p<0.0005$ ). Median OS was also longer for the patients in the combination therapy arm (29.4 vs. 12.8 months; HR: 0.33; 95\% CI: 0.12-0.91; $p=0.025)$. Patients receiving combination therapy had a higher incidence of grade 3-4 toxicities; however, the authors attributed this to the longer duration that they received protocol treatment compared with patients in the control arm.

Hendlisz et al subsequently published a multicenter study of patients with liver-only colorectal metastases treated with fluorouracil with or without resin microsphere radioembolization. ${ }^{11}$ Forty-six patients were randomized, and 44 were eligible for analysis, of whom 21 received combination therapy and 23 received chemotherapy alone. Most patients had been heavily pretreated with a regimen that included antiepidermal growth factor receptor therapy and irinotecan.

The time to liver progression (TTLP) was the primary endpoint and was available for 41 patients. TTLP was longer 
in the combination therapy arm compared with the control arm (5.5 vs. 2.1 months, HR: 0.38; 95\% CI: 0.20-0.72; $p=0.003)$. Median TTP at any site was longer in the combination therapy arm compared with the control arm (4.5 vs. 2.1 months; HR: $0.51 ; 95 \% \mathrm{CI}: 0.28-0.94 ; p=0.03)$. There was no statistical difference in median OS (10.0 vs. 7.3 months, HR: 0.92; 95\% CI: 0.47-1.78; $p=0.80$ ). However, 10 patients (43\%) from the control arm crossed over and received radioembolization, in addition to $70 \%$ of patients in the fluorouracil-only arm who received additional nonradioembolization therapies, which likely confounded the survival data. Grade 3-4 toxicity occurred in six patients receiving chemotherapy alone and one patient receiving combination therapy $(p=0.10)$.

The authors concluded that the randomized trial not only met its primary endpoint demonstrating that radioembolization in combination of intravenous infusion of fluorouracil significantly prolongs the time of both local and distant disease progression.

In 2017, Wasan et al published the combined results of three multicenter, multinational studies (FOXFIRE, SIRFLOX, and FOXFIRE-global) of combination radioembolization with first-line systemic chemotherapy for patients with unresectable colorectal liver metastases. ${ }^{12}$ A total of 1,103 patients were randomized to receive FOLFOX chemotherapy (fluorouracil, leucovorin, oxaliplatin) alone or in combination with resin microsphere radioembolization. In the combination therapy arm, radioembolization was performed concurrently with the first or second cycle of chemotherapy. The primary endpoint was OS.

A total of 549 patients were randomized to the control arm and 554 patients were randomized to the combination therapy arm. The study demonstrated no difference in median OS between the two groups: 22.6 months (95\% Cl: 21.0-24.5) for patients receiving radioembolization plus chemotherapy compared with 23.3 months (95\% CI: 21.8-24.7) for patients receiving chemotherapy alone (HR: 1.04, 95\% CI: 0.90-1.19; $p=0.61)$. However, subgroup analysis did show a significant benefit for combination therapy in patients with right-sided primary tumors (HR: $0.67,95 \% \mathrm{CI}$ : 0.48-0.92).

An objective response was achieved in a higher percentage of patients in the combination therapy arm (72 vs. 63\%) with significantly higher odds of achieving an objective response with combination therapy (OR: 1.78, 95\% CI: $1.37-2.31, p<0.0001)$. However, this did not translate into a higher rate of hepatic resection in the combination therapy arm (17 vs $16 \%$ ), and there was no significant difference in the odds of receiving hepatic resection between the two groups (OR: $1.07,95 \% \mathrm{CI}$ : $0.78-1.48, p=0.67$ ).

The authors suggested that the lack of benefit with the addition of radioembolization to systemic chemotherapy might be explained by the high proportion of patients who developed disease progression outside of the liver, regardless of whether the patients had extrahepatic disease at the time of randomization.

Twenty-five percent of the study population had rightsided primary tumors. The authors noted that several studies have shown worse survival outcomes for patients with rightsided primary tumors and may benefit less from the standard therapies for metastatic colorectal cancer. The finding of a survival benefit in this subgroup warrants further evaluation in future studies.

The EPOCH study is a multinational trial investigating the addition of glass microsphere radioembolization to secondline chemotherapy for patients with colorectal liver metastases who progressed during or after first-line chemotherapy. Patient enrollment concluded in October 2018. Maximum enrollment was set to 420 patients, and the study was designed to detect a 2.5-month increase in PFS in the combination therapy group. Results have not yet been released. $^{13}$

\section{Metastatic Pancreatic Adenocarcinoma}

Gibbs et al published the results of a phase 2 trial evaluating the combination of resin microspheres with systemic chemotherapy in patients with liver-dominant metastatic pancreatic adenocarcinoma. ${ }^{14}$ Patients received a single session of radioembolization during cycle 1 of intravenous 5-fluorouracil (5-FU). The primary endpoint was PFS in the liver, with a threshold median of $\geq 16$ weeks of PFS to be considered clinically significant.

The authors reported a PFS of 5.2 months in the liver and 4.4 months at any site. Median OS was 5.5 months for the entire cohort (range: 1.4-19.5 months). Patients with a resected primary demonstrated a prolonged PFS as well as a longer median OS compared with those with the primary in situ (PFS: 7.8 vs. 3.4 months, $p=0.017$; OS: 13.6 vs. 4.2 months, $p=0.015$ ).

Radioembolization-related toxicities were similar to that of prior studies of radioembolization, including mild-tomoderate abdominal pain, nausea, and transient changes in liver biochemistries. Toxicities associated with 5-FU were also similar to toxicities reported in other trials in which radioembolization had been combined with 5 -FU. ${ }^{10,11}$

The authors concluded that patients with no extrahepatic disease are likely to derive the greatest benefit. In this trial, the best results occurred in the two patients who had liveronly disease, whereas the worst outcomes occurred in the four patients with the primary tumor in situ in addition to extrahepatic disease.

\section{Conclusion}

Several trials have investigated use of combination radioembolization with systemic therapies. Prospective phase 2 and phase 3 clinical trials provide the highest levels of evidence of combination therapy, including protocolized timing of administration of the two treatment modalities, as well as prospectively collected safety and toxicity data. With the advent of entirely new classes of systemic agents, particularly molecularly targeted therapies and immunotherapies, defining the role of combination therapy with radioembolization through prospective clinical trials is increasingly important. 


\section{Conflict of Interest}

The author reports personal fees from Boston Scientific, Merck \& Co., Inc, and Bayer, outside the submitted work.

\section{References}

1 Kulik L, Vouche M, Koppe S, et al. Prospective randomized pilot study of $\mathrm{Y} 90+/$-sorafenib as bridge to transplantation in hepatocellular carcinoma. J Hepatol 2014;61(02):309-317

2 Yao FY, Ferrell L, Bass NM, et al. Liver transplantation for hepatocellular carcinoma: expansion of the tumor size limits does not adversely impact survival. Hepatology 2001;33(06):1394-1403

3 Ricke J, Klümpen $\mathrm{HJ}$, Amthauer $\mathrm{H}$, et al. Impact of combined selective internal radiation therapy and sorafenib on survival in advanced hepatocellular carcinoma. J Hepatol 2019;71(06): 1164-1174

4 Garin E, Rolland Y, Edeline J, et al. Personalized dosimetry with intensification using 90Y-loaded glass microsphere radioembolization induces prolonged overall survival in hepatocellular carcinoma patients with portal vein thrombosis. J Nucl Med 2015;56(03):339-346

5 Hermann AL, Dieudonné A, Ronot M, et al; SARAH Trial Group. Relationship of tumor radiation-absorbed dose to survival and response in hepatocellular carcinoma treated with transarterial radioembolization with ${ }^{90} \mathrm{Y}$ in the SARAH study. Radiology 2020; 296(03):673-684

6 Zhan C, Ruohoniemi D, Shanbhogue KP, et al. Safety of combined yttrium-90 radioembolization and immune checkpoint inhibitor immunotherapy for hepatocellular carcinoma. J Vasc Interv Radiol 2020;31(01):25-34

7 Edeline J, Touchefeu Y, Guiu B, et al. Radioembolization plus chemotherapy for first-line treatment of locally advanced intrahepatic cholangiocarcinoma: a phase 2 clinical trial. JAMA Oncol 2020;6(01):51-59
8 Choi H, Charnsangavej C, de Castro Faria S, et al. CT evaluation of the response of gastrointestinal stromal tumors after imatinib mesylate treatment: a quantitative analysis correlated with FDG PET findings. AJR Am J Roentgenol 2004;183(06):1619-1628

9 Gray B, Van Hazel G, Hope M, et al. Randomised trial of SIRSpheres plus chemotherapy vs. chemotherapy alone for treating patients with liver metastases from primary large bowel cancer. Ann Oncol 2001;12(12):1711-1720

10 Van Hazel G, Blackwell A, Anderson J, et al. Randomised phase 2 trial of SIR-Spheres plus fluorouracil/leucovorin chemotherapy versus fluorouracil/leucovorin chemotherapy alone in advanced colorectal cancer. J Surg Oncol 2004;88(02):78-85

11 Hendlisz A, Van den Eynde M, Peeters M, et al. Phase III trial comparing protracted intravenous fluorouracil infusion alone or with yttrium-90 resin microspheres radioembolization for liverlimited metastatic colorectal cancer refractory to standard chemotherapy. J Clin Oncol 2010;28(23):3687-3694

12 Wasan HS, Gibbs P, Sharma NK, et al; FOXFIRE Trial Investigators SIRFLOX Trial Investigators FOXFIRE-Global Trial Investigators. First-line selective internal radiotherapy plus chemotherapy versus chemotherapy alone in patients with liver metastases from colorectal cancer (FOXFIRE, SIRFLOX, and FOXFIRE-Global): a combined analysis of three multicentre, randomised, phase 3 trials. Lancet Oncol 2017;18(09):1159-1171

13 Chauhan N, Mulcahy MF, Salem R, et al. TheraSphere yttrium-90 glass microspheres combined with chemotherapy versus chemotherapy alone in second-line treatment of patients with metastatic colorectal carcinoma of the liver: protocol for the $\mathrm{EPOCH}$ phase 3 randomized clinical trial. JMIR Res Protoc 2019;8(01): e11545

14 Gibbs P, Do C, Lipton L, et al. Phase II trial of selective internal radiation therapy and systemic chemotherapy for liver-predominant metastases from pancreatic adenocarcinoma. BMC Cancer 2015; 15:802 